\section{Strain or plasmid}

\section{$P$. aeruginosa}

\begin{tabular}{|c|c|}
\hline PA01 & Clinical isolate \\
\hline wzm-G01::ISphoA/hah & PA01 wzm knockout \\
\hline wzt-E12::ISphoA/hah & PA01 wzt knockout \\
\hline E2 & Environmental isolate \\
\hline E2-M4 & Pyocin L1 tolerant mutant \\
\hline E2-M11 & Pyocin L1 tolerant mutant \\
\hline P1 & Clinical isolate \\
\hline $\mathrm{P} 2$ & Clinical isolate \\
\hline P3 & Clinical isolate \\
\hline P4 & Clinical isolate \\
\hline P5 & Clinical isolate \\
\hline P7 & Clinical isolate \\
\hline P8 & Clinical isolate \\
\hline P9 & Clinical isolate \\
\hline $\mathrm{P} 10$ & Clinical isolate \\
\hline P11 & Clinical isolate \\
\hline P12 & Clinical isolate \\
\hline P13 & Clinical isolate \\
\hline P14 & Clinical isolate \\
\hline P15 & Clinical isolate \\
\hline P16 & Clinical isolate \\
\hline P17 & Clinical isolate \\
\hline P18 & Clinical isolate \\
\hline P19 & Clinical isolate \\
\hline YH5 & Clinical isolate \\
\hline PA14 & Clinical isolate \\
\hline PA7 & Clinical isolate \\
\hline PA62 & Environmental isolate \\
\hline MSH10 & Environmental isolate \\
\hline MSH3 & Environmental isolate \\
\hline C763 & Clinical isolate \\
\hline C1334 & Clinical mucoid isolate \\
\hline C1426 & Clinical isolate \\
\hline $\mathrm{C} 1433$ & Clinical mucoid isolate \\
\hline $\mathrm{J} 1385$ & Clinical isolate \\
\hline $\mathrm{J} 1532$ & Clinical mucoid isolate \\
\hline
\end{tabular}

\section{Reference/Source Pyocin L1 Sensitvity}

[1]

This study

This study

This study

This study

This study

This study

This study

This study

This study

This study

This study

This study

This study

This study

This study

This study

This study

This study

This study

This study

This study

[4]

[5]

[6]

[3]

[3]

[6]

[6]

[6]

[6]

[7]
$6.25 \mu \mathrm{g} / \mathrm{ml}$

$0.78 \mu \mathrm{g} / \mathrm{ml}$

$2.6 \mathrm{mg} / \mathrm{ml}$ (very weak)

$2.6 \mathrm{mg} / \mathrm{ml}$ (very weak)

$400 \mu \mathrm{g} / \mathrm{ml}$

$400 \mu \mathrm{g} / \mathrm{ml}$

$25 \mu \mathrm{g} / \mathrm{ml}$

$400 \mu \mathrm{g} / \mathrm{ml}$

$0.39 \mu \mathrm{g} / \mathrm{ml}$

$200 \mu \mathrm{g} / \mathrm{ml}$

$50 \mu \mathrm{g} / \mathrm{ml}$

\title{
P. syringae
}

$$
\text { pv. tomato DC3000 }
$$

pv. tomato NCPPB 1107

pv. tomato NCPPB 2563

pv. tomato NCPPB 3160

pv. coronafaciens LMG

isolated from Solanum

lycopersicum

$\mathrm{BCCM}$

isolated from Solanum

lycopersicum

NCPPB

isolated from Solanum

lycopersicum

NCPPB

isolated from Solanum

lycopersicum

NCPPB

isolated from Avena sativa

$$
5060
$$


pv. lachrymans LMG 5456 isolated from Cucumis sativus

pv. maculicola LMG 2208

morsprunorum LMG2222 isolated from Prunus avium

pv. syringae LMG1247

pv. syringae LMG 5082 isolated from Zea mays

pv. syringae LMG 5084 isolated from Pyrus communis
$\mathrm{BCCM}$

$2.6 \mathrm{mg} / \mathrm{ml}$ (very weak)

BCCM

BCCM

$\mathrm{BCCM}$

BCCM

$\mathrm{BCCM}$
$2.6 \mathrm{mg} / \mathrm{ml}$ (very weak)

$2.6 \mathrm{mg} / \mathrm{ml}$ (very weak)

$2.6 \mathrm{mg} / \mathrm{ml}$ (very weak)

$2.6 \mathrm{mg} / \mathrm{ml}$ (very weak)

\section{P. carotovorum subsp. carotovorum}

LMG 2410

Isolated from Cucumis sativus

$\mathrm{BCCM}$

LMG 2412

Isolated from Hyacinthus orientalis $\mathrm{BCCM}$

LMG 2442

Isolated from Brassica oleracea

BCCM

LMG 2444

Isolated from Solanum tuberosum

(tuber soft rot)

$\mathrm{BCCM}$

LMG 2913

Isolated from soil

$\mathrm{BCCM}$

\section{P. atrosepticum}

LMG 2374

LMG 2375

LMG 2386

LMG 2391

SCRI 1043

Isolated from Apium graveolens var. dulce

BCCM

Isolated from Solanum tuberosum

(tuber soft rot)

$\mathrm{BCCM}$

Isolated from Solanum tuberosum

(stem rot)

$\mathrm{BCCM}$

Isolated from soil

$\mathrm{BCCM}$

Isolated from Solanum tuberosum

(tuber soft rot)

SCRI

E. coli

DH5 $\alpha$

F-, $\varphi 80$ dlacZ $\Delta$ M15, $\Delta$ (lacZYA-

$\operatorname{argF}) \mathrm{U} 169$, deoR, recA1, endA1, hsdR17(rk',

$\left.m k^{+}\right)$, phoA, supE44, $\lambda^{-}$, thi-

1, gyrA96, relA1

Invitrogen

BL21(DE3)pLysS

$\mathrm{F}^{-}$omp T hsdS B $\left(\mathrm{rB}^{-} \mathrm{mB}^{-}\right)$gal dcm (DE3) pLysS $\left(\mathrm{Cam}^{\mathrm{R}}\right)$

Invitrogen

\section{Plasmids}

pET21a
pETPyoL1

pETPL1
Amp ${ }^{\mathrm{r}}$, cloning/expression vector, $\mathrm{T} 7$ promoter $771 \mathrm{bp}$ xhoI/NdeI fragment in pET21a $828 \mathrm{bp}$ xhol/NdeI fragment in pET21a
Novagen

This study

This study 